\title{
Clinical validity of increased cortical binding of tau ligands of the THK family and PBB3 on PET as biomarkers for Alzheimer's disease in the context of a structured 5-phase development framework
}

\author{
Konstantinos Chiotis ${ }^{1,2}$ - Alessandra Dodich ${ }^{3,4}$ - Marina Boccardi ${ }^{5}$ - Cristina Festari $^{6}$ - Alexander Drzezga ${ }^{7,8,9}$. \\ Oskar Hansson ${ }^{10,11} \cdot$ Rik Ossenkoppele $^{12,13} \cdot$ Giovanni Frisoni $^{5,14} \cdot$ Valentina Garibotto $^{4,15} \cdot$ Agneta Nordberg $^{1,16}$
}

Received: 30 October 2020 / Accepted: 21 February 2021 / Published online: 15 March 2021

(C) The Author(s) 2021

\begin{abstract}
Purpose The research community has focused on defining reliable biomarkers for the early detection of the pathological hallmarks of Alzheimer's disease (AD). In 2017, the Geneva AD Biomarker Roadmap initiative adapted the framework for the systematic validation of oncological biomarkers to $\mathrm{AD}$, with the aim to accelerate their development and implementation in clinical practice. The aim of this work was to assess the validation status of tau PET ligands of the THK family and PBB3 as imaging biomarkers for $\mathrm{AD}$, based on the Biomarker Roadmap methodology.

Methods A panel of experts in AD biomarkers convened in November 2019 at a 2-day workshop in Geneva. The level of clinical validity of tau PET ligands of the THK family and PBB3 was assessed based on the 5-phase development framework before the meeting and discussed during the workshop.

Results PET radioligands of the THK family discriminate well between healthy controls and patients with AD dementia (phase 2; partly achieved) and recent evidence suggests an accurate diagnostic accuracy at the mild cognitive impairment (MCI) stage of the disease (phase 3; partly achieved). The phases 2 and 3 were considered not achieved for PBB3 since no evidence exists about the ligand's diagnostic accuracy. Preliminary evidence exists about the secondary aims of each phase for all ligands.

Conclusion Much work remains for completing the aims of phases 2 and 3 and replicating the available evidence. However, it is unlikely that the validation process for these tracers will be completed, given the presence of off-target binding and the development of second-generation tracers with improved binding and pharmacokinetic properties.
\end{abstract}

This article is part of the Topical Collection on Neurology - Dementia

Agneta Nordberg

agneta.k.nordberg@ki.se

1 Nordberg Translational Molecular Imaging Lab, Division of Clinical Geriatrics, Center for Alzheimer Research, Department of Neurobiology, Care Sciences and Society, Karolinska Institutet, Neo 7th floor, 14183 Stockholm, Sweden

2 Department of Neurology, Karolinska University Hospital, Stockholm, Sweden

3 Center for Neurocognitive Rehabilitation (CeRiN), CIMeC, University of Trento, Trento, Italy

$4 \quad$ NIMTlab-Neuroimaging and Innovative Molecular Tracers Laboratory, University of Geneva, Geneva, Switzerland

5 German Center for Neurodegenerative Diseases (DZNE), Rostock, Germany

6 LANE - Laboratory of Alzheimer's Neuroimaging and Epidemiology, IRCCS Istituto Centro San Giovanni di Dio Fatebenefratelli, Brescia, Italy

7 Faculty of Medicine, University of Cologne, Cologne, Germany
8 German Center for Neurodegenerative Diseases (DZNE), Bonn/ Cologne, Germany

9 Institute of Neuroscience and Medicine (INM-2), Molecular Organization of the Brain, Research Center Jülich, Jülich, Germany

10 Clinical Memory Research Unit, Department of Clinical Sciences Malmö, Lund University, Lund, Sweden

11 Memory Clinic, Skåne University Hospital, Malmö, Sweden

12 Alzheimer Center Amsterdam, Department of Neurology, Amsterdam Neuroscience, Vrije Universiteit Amsterdam, Amsterdam UMC, Amsterdam, Netherlands

13 Department of Clinical Memory Research, Lund University, Lund, Sweden

14 Memory Clinic, University Hospital, Geneva, Switzerland

15 Nuclear Medicine and Molecular Division, Geneva Medical Hospital, Geneva, Switzerland

16 Theme Inflammation and Aging, Karolinska University Hospital, Stockholm, Sweden 
Keywords Alzheimer's disease $\cdot$ Strategic roadmap $\cdot$ Biomarker-based diagnosis $\cdot$ Tau PET $\cdot$ THK $\cdot$ PBB3

\section{Introduction}

In the past few years, there has been a boost in the development of imaging and fluid biomarkers for tau pathology, one of the neupathological hallmarks of Alzheimer's disease (AD). Regarding imaging, a wide variety of PET ligands for tau was developed and an extensive amount of data from clinical studies has been published internationally from different research groups in a relatively short period of time (for a detailed review see [1]). However, whether these results support the validity of those tau biomarkers for use in a clinical setting remains to be assessed systematically. Such an assessment is of particular interest at the moment, given the recent US Food and Drug Administration (FDA) approval for clinical use of one of the developed ligands targeting tau pathology (i.e., AV-1451, aka Flortaucipir or Tauvid).

In 2017, a methodological 5-phase framework for the systematic assessment of biomarker validation has been imported from oncology [2] and adapted to AD [3]. Within this Biomarker Roadmap initiative, we had assessed the clinical validation status of all wellconsolidated biomarkers at the time for a specific context of use, namely improved clinical diagnosis in patients presenting to memory clinics with mild cognitive impairment (MCI) [4]. These biomarkers included the episodic memory assessment [5], cerebrospinal fluid (CSF) measures [6], medial temporal atrophy [7], ${ }^{18} \mathrm{~F}$-fluorodeoxyglucose (FDG) PET [8], amyloid- $\beta$ ligands on PET [9], and ${ }^{123}$ Iioflupane brain single-photon emission tomography and ${ }^{123}$ I-metaiodobenzylguanidine (MIBG) cardiac scintigraphy [10].

The present systematic review aims to apply the developed methodological 5-phase framework of the Biomarker Roadmap initiative-in its 2020 update that aimed to align it with the current research criteria [11] and accommodate tau biomarkers (Boccardi et al., in this Issue doi: https://doi.org/10.1007/s00259-02005120-2) - for assessing the validation status of the tau PET ligands of the THK family (i.e., THK5117, THK5317, THK5351) and PBB3 for clinical use. Separate reviews assess the clinical validity of the other available tau PET ligands and measures of tau in CSF and plasma (Wolters et al.; Leuzy et al.; Bischof et al.; Ashton et al., in this Issue, doi: https://doi.org/10. 1007/s00259-020-05118-w; https://doi.org/10.1007/ s00259-020-05156-4).

\section{Methods}

\section{Target}

This literature review investigates the clinical validation status of tau PET ligands as AD biomarkers, in accordance with the Biomarker Roadmap initiative [3, 4] 2020 update (Boccardi et al., in this Issue). The context of biomarker use entails the accurate diagnosis of patients with MCI referred to memory clinics for cognitive complaints, which are attributed to a possible sporadic, and not autosomal dominant, dementing neurodegenerative disorder. The studies of ligands of the THK family (i.e., THK5117, THK5317, THK5351) and PBB3 that were eligible for this review used as reference standard for the biomarker-based diagnosis either AD histopathological examination when available, amyloid- $\beta$ biomarker positivity, or development of incidental AD dementia during a follow-up interval of at least 2 years. Thus, eligible studies for assessing the clinical validity of the biomarker were both prospective longitudinal and cross-sectional designs. For the aims of the review, only ligands of the THK family and PBB3 were taken into consideration. The evidence for other ligands and the available tau bio-fluid markers is discussed elsewhere (Wolters et al.; Leuzy et al.; Bischof et al.; Ashton et al., in this issue).

\section{Glossary}

\section{Neuropathological diagnosis of Alzheimer's disease}

The definite diagnosis of AD is based on the presence of extracellular amyloid- $\beta$ plaques and aggregates of hyperphosphorylated tau in neurofibrillary tangles in the brain of the affected individuals, independently from the clinical expression of cognitive symptoms. The presence of aggregates of amyloid- $\beta$ and tau is often associated to the AD-pattern of medial temporal and temporoparietal neurodegeneration.

\section{Clinical diagnosis of Alzheimer's disease dementia}

According to the classical criteria, as defined by the National Institute of Neurological and Communicative Disorders and Stroke and the Alzheimer's disease and Related Disorders Association (NINCDS-ADRDA), AD can be diagnosed in the clinic at the dementia stage of the disease with two levels of certainty, namely possible and probable AD [12]. Notably, because of the imperfect accuracy of purely clinical criteria, a 
percentage of cases with a clinical diagnosis of $\mathrm{AD}$ might suffer from non-AD pathology since the diagnostic gold standard remains the histopathological examination. The most recent research criteria support that the use of biomarkers, especially those targeting amyloid- $\beta$ pathology, could increase the diagnostic certainty that the basis of the clinical dementia syndrome is the $\mathrm{AD}$ pathophysiological process (i.e., $\mathrm{AD}$ dementia diagnosis; $[13,14])$.

\section{Mild cognitive impairment}

This diagnosis refers to individuals with an acquired objective cognitive impairment but without, or with subtle functional disability. Representing a clinical syndrome, it encompasses cases progressing to $\mathrm{AD}$ (about $50 \%$ ) or non- $\mathrm{AD}$ dementia (about 10-15\%; [15-17]), and cases that are not deteriorating further cognitively over time (about 35-40\%). MCI cases positive to $\mathrm{AD}$ biomarkers can be defined as prodromal $\mathrm{AD}$ or $\mathrm{MCI}$ due to $\mathrm{AD}$ based on research diagnostic criteria $[14,18]$. The diagnosis of $\mathrm{AD}$ at the MCI stage represents the focus of the present review.

\section{Non-AD neurodegenerative disease}

This term refers to the large spectrum of neurodegenerative disorders considered for the differential diagnosis (e.g., the variants of frontotemporal lobar degeneration, dementia with Lewy bodies, hippocampal sclerosis, limbic-predominant agerelated transactive response DNA-binding protein of $43 \mathrm{kDa}$ (aka TDP-43) encephalopathy, primary age-related tauopathy (aka PART), argyrophilic grain disease).

\section{Conceptual framework}

Details on the conceptual framework have been extensively discussed [3]. For each phase/aim, different strings were used to detect relevant studies, which were selected following PRISMA guidelines (see Online Resource for strings and PRISMA results). For all included studies, relevant information about study design, methods, and results was recorded.

\section{Phase 1}

This phase includes preclinical exploratory studies on the rational for using tau PET ligands of the THK family and PBB3 for detecting tau pathology in AD. The gold-standard for phase 1 studies is histopathological examination.

\section{Phase 2}

Phase 2 studies investigate the diagnostic accuracy of tau PET ligands of the THK family and PBB3 to distinguish patients with clinical diagnosis of AD from controls. Phase 2 studies are meant to define the clinical assay, to allow reliable assessment, and to identify the effect of confounders affecting the threshold for positivity in both patients and controls (e.g., age, gender, apolipoprotein $\varepsilon 4$ status, education or comorbidities).

\section{Phase 3}

Phase 3 studies assess the biomarker ability to detect the disease at its earliest possible stage, namely MCI for this specific effort, in well-controlled experimental samples. Phase 3 studies aim to define criteria for positivity, to compare the diagnostic performance with other biomarkers, and to assess the diagnostic value of combinations of biomarkers, in view of defining a biomarkerbased algorithm. These are normally prospective, longitudinal studies that distinguish between MCI individuals that deteriorate cognitively over time in the $\mathrm{AD}$ continuum (i.e., $\mathrm{AD}$ dementia) from those remaining cognitively stable or being diagnosed with other non-AD neurodegenerative diseases at follow-up. However, given the relative absence of such studies in the field of tau PET imaging to date, we even assessed at this phase separately studies with MCI patients, where the gold standard was not the follow-up assessment but the cross-sectional amyloid- $\beta$ biomarker status of the patients, in accordance with the existing research criteria. Nonetheless, the cross-sectional amyloid- $\beta$ biomarker status represents only a construct validity for this phase and the level of evidence that it provides is lower than the follow-up studies, given the inherent limitations of validating a new biomarker to a non-perfect existing clinical marker [19].

\section{Phase 4}

Phase 4 studies assess the performance of tau PET ligands of the THK family and PBB3 in representative patient cohorts from memory clinics. The biomarker itself is used to support a clinical diagnosis to patients with MCI who are subsequently treated based on this tau PET-supported diagnosis. They are meant to quantify the benefit of tau PET-based early detection, and of practical feasibility and protocol compliance. Preliminary evidence about costs is an additional aim, in view of dedicated studies in phase 5 . 


\section{Phase 5}

Phase 5 studies evaluate the impact of the tau PET-based diagnosis on society (e.g., cost-effectiveness relative to clinically meaningful outcomes).

\section{Evidence assessment}

The fulfillment of each validation step from phase 1 to phase 5 has been assessed consistently with the 2017 Biomarker Roadmap initiative [3], update 2020 (Boccardi et al., in this issue). As such, primary and secondary aims for each phase were rated as follows: fully achieved, partly achieved, preliminary evidence, not achieved or unsuccessful, as defined below. To facilitate the assessment and make it transparent to the readers, the data used to define the degree of fulfillment for each aim are reported and summarized in tables accessible online (see Online Resource).

Fully achieved: available scientific evidence, successfully replicated in properly powered and well-designed studies.

Partly achieved: the available evidence is not sufficiently replicated, or samples are not adequately powered, or studies are faulted with major methodological limitations.

Preliminary evidence: only preliminary evidence is available.

Not achieved: studies are not yet performed at the time of the review.

Unsuccessful: available scientific evidence shows a failure for the biomarker in achieving the aim. Findings in the subsequent Biomarker Roadmap phases should be interpreted with caution.

\section{Papers search and selection}

For each phase, we performed systematic literature searches for studies investigating the previously mentioned aims. We searched PubMed and Embase databases for relevant studies. The search was conducted on 16.05.2020. General search strings ("THK-5117” OR “THK-5317” OR “THK-5351" OR “THK5117” OR “THK5317” OR “THK5351” OR "PBB3") were used to identify articles about the relevant tau PET ligands, which were combined with specific search strings for every phase, as detailed in the Online Resource. Only articles written in English were included. These searches were supplemented by the admission of relevant evidence based on personal knowledge or from the reference lists of pertinent articles. After excluding the duplicates, all titles identified by search strategies were assessed for relevance based on their abstract independently by two reviewers, the authors $\mathrm{KC}$ and $\mathrm{CF}$. All resulting records were assessed for eligibility in their full text by the same authors (KC, CF). The reasons for exclusion and the number of finally retained papers for each phase/aim are reported according to the PRISMA guidance [20] on the Online Resource (https:// drive.switch.ch/index.php/s/4reUTSuqNZHyIC8).

\section{Current clinical validity of tau PET THK and PBB3 imaging}
a) Systematic review searches

Studies identified for each phase/aim are reported in the respective PRISMA flow charts (Online Resource).

b) Clinical validity of ligands of the THK family and PBB3

Figures 1 and 2 summarize the current state of tau PET ligands of the THK family and PBB3, respectively, as per our methodological framework.

\begin{tabular}{|c|c|c|c|c|c|c|c|c|c|}
\hline \multicolumn{4}{|c|}{ Analytical validity } & \multicolumn{4}{|c|}{ Clinical Validity } & \multirow{2}{*}{\multicolumn{2}{|c|}{$\begin{array}{c}\text { Clinical Utility } \\
\text { Phase 5 } \\
\text { Implementation }\end{array}$}} \\
\hline \multirow{2}{*}{\multicolumn{2}{|c|}{$\begin{array}{c}\text { Phase } 1 \\
\text { Specimens } \\
\text { Primary aim } \\
\end{array}$}} & \multicolumn{2}{|c|}{$\begin{array}{c}\text { Phase } 2 \\
\text { Clinical assay }\end{array}$} & \multicolumn{2}{|c|}{$\begin{array}{c}\text { Phase } 3 \\
\text { Set clinical use }\end{array}$} & \multicolumn{2}{|c|}{$\begin{array}{l}\text { Phase } 4 \\
\text { Real world performance }\end{array}$} & & \\
\hline & & Primary aim & Secondary aims & Primary aims & Secondary aims & Primary aim & Secondary aims & Primary aim & Secondary aims \\
\hline \multirow{2}{*}{\multicolumn{2}{|c|}{ Leads }} & \multirow{2}{*}{$\begin{array}{c}\text { Accuracy } \\
\text { AD/HC }\end{array}$} & $\begin{array}{c}\text { Assay } \\
\text { definition }\end{array}$ & \multirow{2}{*}{$\begin{array}{l}\text { Accuracy } \\
\mathrm{MCl} / \mathrm{HC}\end{array}$} & $\begin{array}{l}\text { Impact of } \\
\text { covariates }\end{array}$ & \multirow{4}{*}{$\begin{array}{l}\text { Correct } \\
\text { diagnoses }\end{array}$} & $\begin{array}{l}\text { Predictive } \\
\text { features }\end{array}$ & \multirow{4}{*}{$\begin{array}{l}\text { Impact on } \\
\text { relevant } \\
\text { outcomes }\end{array}$} & $\begin{array}{c}\text { Cost } \\
\text { assessment }\end{array}$ \\
\hline & & & $\begin{array}{c}\text { Ante } \\
\text { mortem/ } \\
\text { autopsy }\end{array}$ & & $\begin{array}{l}\text { Compare } \\
\text { markers }\end{array}$ & & $\begin{array}{l}\text { Feasibility \& } \\
\text { Compliance }\end{array}$ & & \begin{tabular}{|c|} 
Compliance \\
across \\
settings \\
\end{tabular} \\
\hline \multicolumn{3}{|c|}{ Achievement } & Covariates in & \multirow{3}{*}{$\begin{array}{l}\text { Criteria for } \\
\text { positivity }\end{array}$} & Combine & & $\begin{array}{l}\text { Preliminary } \\
\text { impact \& }\end{array}$ & & Compare \\
\hline Full & Partial & Preliminary & & & & & costs & & \\
\hline $\begin{array}{l}\text { Not } \\
\text { chieved }\end{array}$ & $\begin{array}{l}\text { Not } \\
\text { applicarble }\end{array}$ & Unsuccessful & $\begin{array}{c}\text { Covariates in } \\
A D\end{array}$ & & $\begin{array}{l}\text { Testing } \\
\text { Interval }\end{array}$ & & $\begin{array}{l}\text { Monitor false } \\
\text { negatives }\end{array}$ & & \\
\hline
\end{tabular}

Fig. 1 Synopsis of the clinical validity of tau PET ligands of the THK family as adapted from an oncology framework [2, 4] 


\begin{tabular}{|c|c|c|c|c|c|c|c|c|c|}
\hline \multicolumn{4}{|c|}{ Analytical validity } & \multicolumn{4}{|c|}{ Clinical Validity } & \multirow{2}{*}{\multicolumn{2}{|c|}{$\begin{array}{c}\text { Clinical Utility } \\
\text { Phase } 5 \\
\text { Implementation }\end{array}$}} \\
\hline \multirow{2}{*}{\multicolumn{2}{|c|}{$\begin{array}{c}\text { Phase } 1 \\
\text { Specimens }\end{array}$}} & \multicolumn{2}{|c|}{$\begin{array}{c}\text { Phase } 2 \\
\text { Clinical assay }\end{array}$} & \multicolumn{2}{|c|}{$\begin{array}{c}\text { Phase } 3 \\
\text { Set clinical use }\end{array}$} & \multicolumn{2}{|c|}{$\begin{array}{c}\text { Phase } 4 \\
\text { Real world performance }\end{array}$} & & \\
\hline & & Primary aim & Secondary aims & Primary aims & Secondary aims & Primary aim & Secondary aims & Primary aim & Secondary aims \\
\hline \multirow{2}{*}{\multicolumn{2}{|c|}{ Leads }} & \multirow{2}{*}{$\begin{array}{l}\text { Accuracy } \\
\text { AD/HC }\end{array}$} & $\begin{array}{c}\text { Assay } \\
\text { definition }\end{array}$ & \multirow{2}{*}{$\begin{array}{l}\text { Accuracy } \\
\mathrm{MCl} / \mathrm{HC}\end{array}$} & $\begin{array}{l}\text { Impact of } \\
\text { covariates }\end{array}$ & \multirow{5}{*}{$\begin{array}{c}\text { Correct } \\
\text { diagnoses }\end{array}$} & $\begin{array}{l}\text { Predictive } \\
\text { features }\end{array}$ & \multirow{5}{*}{$\begin{array}{l}\text { Impact on } \\
\text { relevant } \\
\text { outcomes }\end{array}$} & $\begin{array}{c}\text { Cost } \\
\text { assessment }\end{array}$ \\
\hline & & & $\begin{array}{c}\text { Ante } \\
\text { mortem/ } \\
\text { autopsy }\end{array}$ & & $\begin{array}{l}\text { Compare } \\
\text { markers }\end{array}$ & & $\begin{array}{l}\text { Feasibility \& } \\
\text { Compliance }\end{array}$ & & $\begin{array}{c}\text { Compliance } \\
\text { across } \\
\text { settings }\end{array}$ \\
\hline \multirow{2}{*}{\multicolumn{3}{|c|}{ Achievement }} & Covariates in & \multirow{4}{*}{$\begin{array}{l}\text { Criteria for } \\
\text { positivity }\end{array}$} & Combine & & Preliminary & & Compare \\
\hline & & & $\mathrm{HC}$ & & markers & & impact \& & & protocols \\
\hline Full & Partial & Preliminary & & & & & & & \\
\hline $\begin{array}{l}\text { Not } \\
\text { chieved }\end{array}$ & $\begin{array}{l}\text { Not } \\
\text { applicable }\end{array}$ & Unsuccessful & $\begin{array}{c}\text { Covariates in } \\
A D\end{array}$ & & $\begin{array}{l}\text { Testing } \\
\text { Interval }\end{array}$ & & $\begin{array}{c}\text { Monitor false } \\
\text { negatives }\end{array}$ & & \\
\hline
\end{tabular}

Fig. 2 Synopsis of the clinical validity of PBB3 PET as adapted from an oncology framework [2, 4]

\section{Phase 1: preclinical exploratory studies}

\section{Primary aim: to identify leads for potentially useful biomarkers}

PET ligands of the THK family (i.e., THK5117, THK5317, THK5351) and PBB3 have shown in vitro high affinity in the nanomolar range for a wide span of different conformations of tau aggregates, and selectivity for tau over amyloid- $\beta$ aggregates [21-27]. When autoradiography in brain tissue was performed, the binding pattern of those ligands resembled closely the binding pattern of standard tau-specific antibodies. All ligands showed pre-clinically favorable pharmacokinetics, with the enantiomerically pure most recent ligands of the THK family (i.e., THK5317, THK5351) showing improved properties compared to their racemic forms. Both the ligands of the THK family and PBB3 have shown evidence of binding to non-tau targets (i.e., off-target binding) predominantly in the basal ganglia and thalamus for the THK ligands, and the basal ganglia and vascular structures (e.g., choroid plexus, dural venous sinuses) for PBB3 (for a detailed review see [1, 28]). This aim was considered fully achieved for the ligands of the THK family and PBB3.

\section{Phase 2: clinical assay development for Alzheimer's disease pathology}

Phase 2: primary aim: to estimate true positive and false positive rates or receiving operating characteristics curves (ROC) for the assay and to identify the discrimination accuracy between subjects with and without the disease

Three small-scale studies ( $n=10-18)$ have assessed the accuracy of the ligands of the THK family in discriminating between clinically diagnosed AD patients and healthy controls (HCs). For THK5117, an effect size of Cohen's $d=3.05$ was reported [22]. In agreement to the latter study, for THK5317 and THK5351, accuracies as high as $99 \%$ and $96 \%$ were reported, respectively [29, 30]. The reported accuracies were dependent of the region of interest selected for each study, with areas of the temporal cortex being consistently reported as having the highest accuracy in both studies. No studies report formally the accuracy of ligands of the THK family to discriminate between clinically diagnosed $\mathrm{AD}$ patients and non-AD neurodegenerative disease although the evidence so far points towards a different regional pattern of ligand for both THK5317 and THK5351 binding in AD compared to other neurodegenerative diseases [29, 31-46].

To the best of our knowledge, no study has yet attempted to assess the accuracy of PBB3, although the evidence so far imply a good accuracy for discrimination of HCs and clinically diagnosed $\mathrm{AD}$ patients and a different regional pattern of binding for non-AD neurodegenerative diseases [27, 47-52].

Given the consistent findings of the small-scale studies, this aim was considered partly achieved for ligands of the THK family and not achieved for PBB3. We underline however that phase 2 is aimed to demonstrate that the assay does detect the anomaly of interest, thus performing studies using pathology as gold standard is of paramount importance and should be considered a short-term priority.

Phase 2: secondary aim 1: to optimize procedures for performing the assay and to assess the reproducibility of the assay within and between laboratories

The binding of THK5317 and THK5351 has shown strong correlation when the ligands were injected in the same individuals [53]. Low test-retest variability for THK5317, absence of known brain-penetrating metabolites for THK5351, and robust quantification of the binding of both ligands were reported even with simplified reference region-based approaches with or without the use of structural imaging for region of interest identification [24, 
29, 30, 53-59]. The effect of partial volume effect correction methods in the binding quantification remains unclear for THK5351, while for THK5317 partial volume effect correction offers better discrimination between diagnostic groups in small regions of interest (e.g., hippocampus, anterior cingulate gyrus) $[29,60]$.

For PBB3 a radiolabeled metabolite, which crosses the blood-brain barrier, has been identified [61, 62], although a robust quantification of the ligand binding could be achieved with various reference region-based approaches, despite the metabolite signal $[63,64]$.

Overall, this aim was considered as partly achieved for ligands of the THK family and PBB3.

Phase 2: secondary aim 2: to assess the consistency of the ante-mortem binding of the ligand and the histopathological measurements of tau pathology

For ligands of the THK family, two independent studies have assessed the consistency of the ligand binding in vivo with histopathological evidence of tau. Harada et al. reported in a single AD case, which was assessed in vivo with THK5351 PET imaging and post-mortem with histopathology, that the THK5351 binding was associated with both the loads of tau pathology and monoamine oxidase $\mathrm{B}$ (MAO-B) enzyme; off-target binding to MAO-B has been identified by translational studies for several of the developed tau PET ligands [65]. On the contrary, Leinonen et al. used a different design and assessed the agreement of in vivo THK5317 binding and histopathological evidence of tau in biopsy material of patients with normal pressure hydrocephalus, without a clinical diagnosis of $\mathrm{AD}$ [66]. The authors reported no agreement between the two, although they acknowledged several limitations that this design raises. Given the major limitations of both studies, only preliminary evidence exists for ligands of the THK family, while no studies exist for PBB3 and therefore the aim was considered as not achieved for this ligand.

Phase 2: secondary aim 3: to assess covariates (such as gender, age) associated with biomarker status or level in control subjects

To the best of our knowledge, no study has yet assessed the impact of covariates in the binding levels of control subjects for ligands of the THK family. Regarding PBB3, evidence suggests a higher binding of the ligand with higher age and lower educational attainment [47]. This aim was considered partly achieved for PBB3, based on evidence of one study addressing the research question, and not achieved for ligands of the THK family.
Phase 2: secondary aim 4: to assess covariates (such as gender and age) associated with biomarker status or level in diseased subjects

The impact of covariates such as gender and age has not yet been assessed for ligands of the THK family in AD patients. However, different AD clinical syndromes were associated with a different regional pattern of binding for the ligand THK5351 [67, 68], which might bias the individual biomarker status if the same regions of interest are used for all patients irrespective of the clinical symptomatology.

For PBB3, evidence suggests a lower binding of the ligand with higher age and higher educational attainment $[47,56]$. The impact of different AD clinical syndromes has not been assessed for PBB3.

In summary, preliminary evidence exists for ligands of the THK family, as can be derived from studies on AD clinical syndromes, while the aim was considered partly achieved for PBB3, based on results of one study investigating this research question.

\section{Phase 3: prospective longitudinal repository studies}

Phase 3: primary aim 1: to evaluate the biomarker ability in the detection of the disease at the earliest clinical stage ( $M C l$ due to $A D$ ) using conversion to $A D$ dementia as the reference standard

A single small-scale monocentric study has, so far, investigated the accuracy of THK5317 in discriminating between amyloid- $\beta$ positive cognitively impaired patients that will remain cognitively stable (considered being non-AD-related cognitive impairment) from those who deteriorated further cognitively to clinical AD dementia, with an average followup interval of 4 years [69]. According to the latter study, the accuracy of THK5317 in temporal regions of interest was excellent, up to $100 \%$. To the best of our knowledge, no study has yet attempted to assess the accuracy of the other ligands of the THK family or PBB3. In summary, this aim was considered partly achieved for ligands of the THK family, based on evidence of one study, while not achieved for PBB3.

Phase 3: primary aim 2: to define criteria for a positive diagnostic test for $M C l$ due to $A D$, in preparation of phase 4

This aim had not been achieved at the time of writing this review.

Phase 3: secondary aim 1: to explore the impact of relevant covariates on the biomarker discrimination abilities at the $\mathrm{MCl}$ stage

This aim had not been achieved at the time of writing this review. 
Phase 3: secondary aim 2: to compare the different biomarkers available to select the most promising ones

The only available study comparing the prognostic accuracy of THK5317 with that of FDG PET, tau assessment in the CSF, clinical atrophy rating on MRI, and neuropsychological measures reported that the THK5317 showed far greater accuracy than all other markers [69]. To the best of our knowledge, no study has yet attempted to assess the accuracy of the other ligands of the THK family or PBB3. This aim was considered partly achieved for ligands of the THK family, based on evidence of one study, while not achieved for PBB3.

Phase 3: secondary aim 3: to develop and validate diagnostic algorithms combining biomarkers for an optimal performance

This aim had not been achieved at the time of writing this review.

Phase 3: secondary aim 4: if repeated testing is needed, to determine a biomarker-testing interval in prevision for phase 4 studies

This aim had not been achieved at the time of writing this review.

\section{Phase 4: prospective diagnostic studies}

The aims of this phase had not been achieved at the time of writing this review.

\section{Phase 5: disease-control studies}

The aims of this phase had not been achieved at the time of writing this review.

\section{Discussion}

With this work, we assessed the clinical validity of the PET ligands of the THK family and PBB3 as biomarkers of brain tauopathy according to the 5-phase framework proposed by the Biomarker Roadmap initiative [4] and its 2020 update (Boccardi et al., in this issue). Ligands of the THK family discriminate well between HCs and patients with clinically diagnosed AD (Phase 2) and recent evidence suggest an accurate diagnostic performance for the THK5317 at the early MCI stage of the disease (phase 3). No formal evidence exists about neither phase 2 nor phase 3 primary aims for PBB3. Little evidence exists, so far, about most secondary aims of phases 2 and 3 for ligands of the THK family and PBB3. No evidence exists for phases 4 and 5 .
Although several reviews about tau PET imaging have been published, no systematic reviews have been performed which would assess the validity of the clinical use of this biomarker in AD. The Biomarker Roadmap initiative assessed originally other biomarkers, but this effort is fully consistent with that validation methodology. This kind of work is necessary to coordinate efforts across independent research groups. Greater awareness of completed steps, research gaps, and priorities based on a sound consensual methodological framework might improve the cost-effectiveness of subsequent validation studies. This work should not be interpreted as an effort to promote the clinical use of the reviewed ligands but rather present in an objective, structured, and validated framework the available evidence for the existing tau biomarkers (tau PET ligands for imaging and tau measures in blood and CSF) in parallel reviews.

The ligands of the THK family have been the only ones from all developed tau PET ligands that were tested longitudinally in a phase 3 study at the time of writing this review [69]. The generalizability of these promising results is, however, subject to several gaps that remain to be filled as research priorities for assessing the clinical validity of the THK ligands: namely, the assessment of (1) the discriminative ability of the assay in pathology confirmed AD and HC samples for phase 2 ; (2) the accuracy of the biomarker to discriminate between $\mathrm{AD}$ and non-AD neurodegenerative disease in adequately powered studies; (3) the consistency of the antemortem and the histopathological measurements of tau pathology, which remains, so far, inconclusive; (4) covariates that are associated with the biomarker status in patients with $\mathrm{AD}$ and HCs; (5) the replicability of the available evidence about the biomarker's diagnostic performance at the MCI stage; (6) criteria of biomarker positivity.

For the purposes of this review, we assessed TH5317 and THK5351 as a whole (THK family) summing up the results obtained from studies using either THK5317 or THK5351 (Fig. 1). This approach was chosen given the high structural similarity of the two ligands and the strong association of the binding of the two when injected in the same individual [53]. However, the main weakness of this generalizability of evidence is the reported differences between ligands in terms of pharmacokinetics and dynamic range (more favorable pharmacokinetics and wider range for THK5351 relatively to THK5317 [53]), and degree of off-target binding (higher for THK5351 relatively to THK5317 [70]) All these factors could affect the comparability of the validity of the two ligands in several aspects of the current framework, although no head-to-head comparisons of diagnostic accuracy have been performed. To address this issue, we reported separately in the text for each aim and subaim which of the two tracers was used to obtain the specific results, in order to allow the reader to critically appraise the available evidence for each ligand. 
For PBB3, phase 2 studies assessing the accuracy of the biomarker to discriminate between clinically diagnosed $\mathrm{AD}$ patients and $\mathrm{HCs}$ or non-AD neurodegenerative diseases are still missing. Those studies, possibly validated by neuropathological assessment, remain the first research priority before one could continue with assessing other research priorities. PBB3 however has a number of limitations, namely (1) the short half-life with need for on-site production of the ligand $\left({ }^{11} \mathrm{C}\right.$-labeled), (2) off-target binding signal, (3) a relatively narrow dynamic range of binding values [56], (4) the existence of brain penetrating metabolites that challenge the quantification of the binding, and (5) the sensitivity of the ligand to photoisomerization that limits the transportation and injection of the ligand in dark conditions.

A fluorinated derivative of PBB3 $\left({ }^{11} \mathrm{C}\right.$-labeled $)$ with somewhat different pharmacological properties has now been developed (i.e., ${ }^{18} \mathrm{~F}$-APN-1607) and is intended to substitute the original ligand [71]. Hopefully, the longer half-life of ${ }^{18} \mathrm{~F}$ would allow the thorough investigation of the ligand and the emergence of replication studies, something that was limited with PBB3 since most of the evidence derives from the original developers of the ligand.

The interaction of tau PET ligands with non-intended targets, the so-called off-target binding, is so far challenging their validity, since several targets have been identified in in vitro, in vivo, and even in silico studies [1,28]. With regard to ligands of the THK family, off-target binding signal has been attributed to binding to MAO-B. MAO-B is an enzyme that catalyzes the de-amination of neurotransmitters, mainly present in subcortical nuclei and it is implicated in a variety of normal and abnormal brain functions. Binding to MAO-B has been the main factor that halted the clinical interest on THK ligands, especially since second generation tau ligands were developed with improved binding and pharmacokinetic properties. The presence of off-target binding (to MAO-B and other non-tau targets) applies not only to ligands of the THK family, but even PBB3 and to the FDA approved ligand AV-1451 (aka, Flortaucipir or Tauvid). However, it is still unclear to what extent this off-target binding contributes to the in vivo signal of the different ligands. Thorough studies assessing the relationship between antemortem PET signal and histopathological measurements of those targets should be prioritized for shedding new light on this point.

Although our approach adhered to a sound methodology, rating degree of achievement for each aim should be based on a more thorough assessment of evidence, including examining various possible sources of bias (e.g., GRADE guidelines [72]). Our Online Resources, reporting data extraction for study features including possible risks of bias, are meant to help this development as a next step forward in a systematic assessment of the validation of $\mathrm{AD}$ biomarkers. Relative to the review results, we admitted studies with clinical reference standard or other evidence of construct validity; however, studies including pathology are still scarce and should be considered a priority for the proper completion of phase 2. Furthermore, another source of bias derives from the admission of evidence solely from peer-reviewed published studies. While the latter was done for ensuring the quality of reported evidence, one should acknowledge that much of the data of proprietary drugs are either published with delay or not published at all.

Acknowledgements This work was supported by the Swiss National Science Foundation (grant n. IZSEZ0_188355), by the Alzheimer's Association, by the OsiriX Foundation, and by the APRA (Association Suisse pour la Recherche sur l'Alzheimer).

We wish to thank the Swedish Foundation for Strategic Research (SSF; RB13-0192), the Swedish Research Council (projects 05817, 2017-0295, and 2017-06086), the Center for Innovative Medicine (CIMED; Region Stockholm and Karolinska Institutet), the Regional Agreement on Medical Training and Clinical Research (ALF) for Region Stockholm, the Swedish Society for Medical Research, the Loo and Hans Osterman Foundation for Medical Research, the Foundation for Geriatric Diseases at Karolinska Institutet, the Magnus Bergvalls Foundation, the Tore Nilson Foundation for Medical Research, the Sigurd and Elsa Golje Memorial, the Eva och Oscar Ahrén Research Foundation Stockholm, the Foundation for Old Servants, the Axel Linder Foundation, the Åhlén Foundation, the Gun and Bertil Stohne Foundation, the Karolinska Institutet Foundations, the Swedish Brain Foundation, the Swedish Alzheimer's Foundation, the Swedish Dementia Association, and the EU FW7 large-scale integrating project INMiND (http://www.uni-muenster.de/INMiND) for financial support. Konstantinos Chiotis was supported by Region Stockholm (clinical postdoctorial appointment).

V. Garibotto was supported by the Swiss National Science Foundation (projects 320030169876 and 320030 185028) and by the Velux foundation (project 11 23 ).

Disclosure statement Open access funding provided by Karolinska Institutet. VG received financial support for research and educational talks through her institution from Siemens Healthineers, GE Healthcare, Life Molecular Imaging, Cerveau Technologies, Roche and Merck. OH has acquired research support (for the institution) from Roche, Pfizer, GE Healthcare, Biogen, Eli Lilly and AVID Radiopharmaceuticals. In the past 2 years, $\mathrm{OH}$ has received consultancy/speaker fees from Biogen, Roche and AC Immune.

Open Access This article is licensed under a Creative Commons Attribution 4.0 International License, which permits use, sharing, adaptation, distribution and reproduction in any medium or format, as long as you give appropriate credit to the original author(s) and the source, provide a link to the Creative Commons licence, and indicate if changes were made. The images or other third party material in this article are included in the article's Creative Commons licence, unless indicated otherwise in a credit line to the material. If material is not included in the article's Creative Commons licence and your intended use is not permitted by statutory regulation or exceeds the permitted use, you will need to obtain permission directly from the copyright holder. To view a copy of this licence, visit http://creativecommons.org/licenses/by/4.0/.

\section{References}

1. Leuzy A, Chiotis K, Lemoine L, Gillberg P-G, Almkvist O, Rodriguez-Vieitez E, et al. Tau PET imaging in neurodegenerative tauopathies — still a challenge. Mol Psychiatry. 2019;24:1112-34. 
2. Pepe MS, Etzioni R, Feng Z, Potter JD, Thompson ML, Thornquist $\mathrm{M}$, et al. Phases of biomarker development for early detection of cancer. J Natl Cancer Inst. 2001;93:1054-61.

3. Boccardi M, Gallo V, Yasui Y, Vineis P, Padovani A, Mosimann U, et al. The biomarker-based diagnosis of Alzheimer's disease. 2lessons from oncology. Neurobiol Aging. 2017;52:141-52.

4. Frisoni GB, Boccardi M, Barkhof F, Blennow K, Cappa S, Chiotis $\mathrm{K}$, et al. Strategic roadmap for an early diagnosis of Alzheimer's disease based on biomarkers. Lancet Neurol. 2017;16:661-76.

5. Cerami C, Dubois B, Boccardi M, Monsch AU, Demonet JF, Cappa SF, et al. Clinical validity of delayed recall tests as a gateway biomarker for Alzheimer's disease in the context of a structured 5phase development framework. Neurobiol Aging. 2017;52:15366.

6. Mattsson N, Lönneborg A, Boccardi M, Blennow K, Hansson O, Geneva Task Force for the Roadmap of Alzheimer's Biomarkers. Clinical validity of cerebrospinal fluid $\mathrm{A} \beta 42$, tau, and phospho-tau as biomarkers for Alzheimer's disease in the context of a structured 5-phase development framework. Neurobiol Aging. 2017;52:196213.

7. Ten Kate M, Barkhof F, Boccardi M, Visser PJ, Jack CR, Lovblad $\mathrm{K}-\mathrm{O}$, et al. Clinical validity of medial temporal atrophy as a biomarker for Alzheimer's disease in the context of a structured 5phase development framework. Neurobiol Aging. 2017;52:167182.e1.

8. Garibotto V, Herholz K, Boccardi M, Picco A, Varrone A, Nordberg A, et al. Clinical validity of brain fluorodeoxyglucose positron emission tomography as a biomarker for Alzheimer's disease in the context of a structured 5-phase development framework. Neurobiol Aging. 2017;52:183-95.

9. Chiotis K, Saint-Aubert L, Boccardi M, Gietl A, Picco A, Varrone A, et al. Clinical validity of increased cortical uptake of amyloid ligands on PET as a biomarker for Alzheimer's disease in the context of a structured 5-phase development framework. Neurobiol Aging. 2017;52:214-27.

10. Sonni I, Ratib O, Boccardi M, Picco A, Herholz K, Nobili F, et al. Clinical validity of presynaptic dopaminergic imaging with 123Iioflupane and noradrenergic imaging with 123I-MIBG in the differential diagnosis between Alzheimer's disease and dementia with Lewy bodies in the context of a structured 5-phase development framework. Neurobiol Aging. 2017;52:228-42.

11. Jack CR, Bennett DA, Blennow K, Carrillo MC, Dunn B, Haeberlein SB, et al. NIA-AA research framework: toward a biological definition of Alzheimer's disease. Alzheimers Dement J Alzheimers Assoc. 2018;14:535-62.

12. McKhann G, Drachman D, Folstein M, Katzman R, Price D, Stadlan EM. Clinical diagnosis of Alzheimer's disease: report of the NINCDS-ADRDA work group under the auspices of Department of Health and Human Services Task Force on Alzheimer's disease. Neurology. 1984;34:939-44.

13. McKhann GM, Knopman DS, Chertkow H, Hyman BT, Jack CR, Kawas $\mathrm{CH}$, et al. The diagnosis of dementia due to Alzheimer's disease: recommendations from the National Institute on AgingAlzheimer's association workgroups on diagnostic guidelines for Alzheimer's disease. Alzheimers Dement J Alzheimers Assoc. 2011;7:263-9.

14. Dubois B, Feldman HH, Jacova C, Hampel H, Molinuevo JL, Blennow K, et al. Advancing research diagnostic criteria for Alzheimer's disease: the IWG-2 criteria. Lancet Neurol. 2014;13: 614-29.

15. Bennett DA, Wilson RS, Schneider JA, Evans DA, Beckett LA, Aggarwal NT, et al. Natural history of mild cognitive impairment in older persons. Neurology. 2002;59:198-205.

16. Rowe CC, Ackerman U, Browne W, Mulligan R, Pike KL, O'Keefe G, et al. Imaging of amyloid beta in Alzheimer's disease with 18F-BAY94-9172, a novel PET tracer: proof of mechanism. Lancet Neurol. 2008;7:129-35.

17. Jack CR, Lowe VJ, Senjem ML, Weigand SD, Kemp BJ, Shiung $\mathrm{MM}$, et al. 11C PiB and structural MRI provide complementary information in imaging of Alzheimer's disease and amnestic mild cognitive impairment. Brain J Neurol. 2008;131:665-80.

18. Albert MS, DeKosky ST, Dickson D, Dubois B, Feldman HH, Fox $\mathrm{NC}$, et al. The diagnosis of mild cognitive impairment due to Alzheimer's disease: recommendations from the National Institute on Aging-Alzheimer's Association workgroups on diagnostic guidelines for Alzheimer's disease. Alzheimers Dement J Alzheimers Assoc. 2011;7:270-9.

19. Dyer SM, Flicker L, Laver K, Whitehead C, Cumming R. The clinical value of fluid biomarkers for dementia diagnosis. Lancet Neurol. 2016;15:1204.

20. Liberati A, Altman DG, Tetzlaff J, Mulrow C, Gøtzsche PC, Ioannidis JPA, et al. The PRISMA statement for reporting systematic reviews and meta-analyses of studies that evaluate health care interventions: explanation and elaboration. J Clin Epidemiol. 2009;62:e1-34.

21. Okamura N, Furumoto S, Harada R, Tago T, Yoshikawa T, FoderoTavoletti M, et al. Novel 18F-labeled arylquinoline derivatives for noninvasive imaging of tau pathology in Alzheimer disease. J Nucl Med Off Publ Soc Nucl Med. 2013;54:1420-7.

22. Harada R, Okamura N, Furumoto S, Furukawa K, Ishiki A, Tomita $\mathrm{N}$, et al. [(18)F]THK-5117 PET for assessing neurofibrillary pathology in Alzheimer's disease. Eur J Nucl Med Mol Imaging. 2015;42: 1052-61.

23. Lemoine L, Saint-Aubert L, Marutle A, Antoni G, Eriksson JP, Ghetti B, et al. Visualization of regional tau deposits using (3)HTHK5117 in Alzheimer brain tissue. Acta Neuropathol Commun. 2015;3:40

24. Harada R, Okamura N, Furumoto S, Furukawa K, Ishiki A, Tomita N, et al. 18F-THK5351: a novel PET radiotracer for imaging neurofibrillary pathology in Alzheimer disease. J Nucl Med Off Publ Soc Nucl Med. 2016;57:208-14.

25. Stepanov V, Svedberg M, Jia Z, Krasikova R, Lemoine L, Okamura N, et al. Development of [11C]/[3H]THK-5351 - a potential novel carbon-11 tau imaging PET radioligand. Nucl Med Biol. 2017;46: 50-3.

26. Tago T, Furumoto S, Okamura N, Harada R, Adachi H, Ishikawa $\mathrm{Y}$, et al. Structure-activity relationship of 2-arylquinolines as PET imaging tracers for tau pathology in Alzheimer disease. J Nucl Med Off Publ Soc Nucl Med. 2016;57:608-14.

27. Maruyama M, Shimada H, Suhara T, Shinotoh H, Ji B, Maeda J, et al. Imaging of tau pathology in a tauopathy mouse model and in Alzheimer patients compared to normal controls. Neuron. 2013;79: 1094-108

28. Lemoine L, Leuzy A, Chiotis K, Rodriguez-Vieitez E, Nordberg A. Tau positron emission tomography imaging in tauopathies: the added hurdle of off-target binding. Alzheimers Dement Amst Neth. 2018;10:232-6.

29. Chiotis K, Saint-Aubert L, Savitcheva I, Jelic V, Andersen P, Jonasson M, et al. Imaging in-vivo tau pathology in Alzheimer's disease with THK5317 PET in a multimodal paradigm. Eur J Nucl Med Mol Imaging. 2016;43:1686-99.

30. Chen J, Li Y, Pirraglia E, Okamura N, Rusinek H, de Leon MJ, et al. Quantitative evaluation of tau PET tracers 18F-THK5351 and 18F-AV-1451 in Alzheimer's disease with standardized uptake value peak-alignment (SUVP) normalization. Eur J Nucl Med Mol Imaging. 2018;45:1596-604.

31. Yoon CW, Jeong HJ, Seo S, Lee S-Y, Suh MK, Heo J-H, et al. 18FTHK5351 PET imaging in nonfluent-agrammatic variant primary progressive aphasia. Dement Neurocognitive Disord. 2018;17:110 9. 
32. Nam G, Jeong HJ, Kang JM, Lee S-Y, Seo S, Seo H-E, et al. 18FTHK5351 PET imaging in the behavioral variant of frontotemporal dementia. Dement Neurocognitive Disord. 2018;17:163-73.

33. Brendel M, Schönecker S, Höglinger G, Lindner S, Havla J, Blautzik J, et al. [18F]-THK5351 PET correlates with topology and symptom severity in progressive supranuclear palsy. Front Aging Neurosci. 2017;9:440.

34. Lee H, Seo S, Lee S-Y, Jeong HJ, Woo S-H, Lee K-M, et al. [18F]THK5351 PET imaging in patients with semantic variant primary progressive aphasia. Alzheimer Dis Assoc Disord. 2018;32:62-9.

35. Shimizu S, Imabayashi E, Takenoshita N, Okamura N, Furumoto S, Kudo Y, et al. Case of progressive supranuclear palsy detected by tau imaging with [18 F]THK-5351 before the appearance of characteristic clinical features. Geriatr Gerontol Int. 2018;18:501-2.

36. Son HJ, Oh JS, Roh JH, Seo SW, Oh M, Lee SJ, et al. Differences in gray and white matter 18F-THK5351 uptake between behavioral-variant frontotemporal dementia and other dementias. Eur J Nucl Med Mol Imaging. 2019;46:357-66.

37. Schaeverbeke J, Evenepoel C, Declercq L, Gabel S, Meersmans K, Bruffaerts R, et al. Distinct [18F]THK5351 binding patterns in primary progressive aphasia variants. Eur J Nucl Med Mol Imaging. 2018;45:2342-57.

38. Kikuchi A, Okamura N, Hasegawa T, Harada R, Watanuki S, Funaki $\mathrm{Y}$, et al. In vivo visualization of tau deposits in corticobasal syndrome by 18 F-THK5351 PET. Neurology. 2016;87:2309-16.

39. Schönecker S, Brendel M, Palleis C, Beyer L, Höglinger GU, Schuh E, et al. PET imaging of astrogliosis and tau facilitates diagnosis of Parkinsonian syndromes. Front Aging Neurosci. 2019;11: 249.

40. Takaya M, Ishii K, Hosokawa C, Saigoh K, Shirakawa O. Tau accumulation in two patients with frontotemporal lobe degeneration showing different types of aphasia using $18 \mathrm{~F}-\mathrm{THK}-5351$ positron emission tomography: a case report. Int Psychogeriatr. 2018;30: 641-6.

41. Takaya M, Ishii K, Kubota I, Shirakawa O. Progression of logopenic aphasia to frontotemporal dementia in an amyloid $\beta$ negative and 18 F-THK-5351-positive patient. Psychogeriatr Off J Jpn Psychogeriatr Soc. 2019;19:399-401.

42. Zhang W, Mao W, Xu E, Chhetri JK, Chan P. Progressive supranuclear palsy presenting with hyperkinetic movement disorder and hemiplegic dystonia: a case report. Int J Neurosci. 2020:14.

43. Jeong HJ, Yoon CW, Seo S, Lee SY, Suh MK, Seo HE, et al. Relationships between $\left[{ }^{18} \mathrm{~F}\right]$-THK5351 retention and language functions in primary progressive aphasia. J Clin Neurol Seoul Korea. 2019;15:527-36

44. Schaeverbeke J, Gabel S, Meersmans K, Bruffaerts R, Liuzzi AG, Evenepoel C, et al. Single-word comprehension deficits in the nonfluent variant of primary progressive aphasia. Alzheimers Res Ther. 2018;10:68.

45. Ishiki A, Harada R, Okamura N, Tomita N, Rowe CC, Villemagne $\mathrm{VL}$, et al. Tau imaging with $[18 \mathrm{~F}] \mathrm{THK}-5351$ in progressive supranuclear palsy. Eur J Neurol. 2017;24:130-6.

46. López-Mora DA, Fernández LA, Lleó A, Blesa R, Camacho V. The added value of tau-PET in the assessment of progressive supranuclear palsy. Clin Nucl Med. 2020;45:e239-40.

47. Shimada H, Kitamura S, Shinotoh H, Endo H, Niwa F, Hirano S, et al. Association between $\mathrm{A} \beta$ and tau accumulations and their influence on clinical features in aging and Alzheimer's disease spectrum brains: a [11C]PBB3-PET study. Alzheimers Dement Amst Neth. 2017;6:11-20.

48. Endo H, Shimada H, Sahara N, Ono M, Koga S, Kitamura S, et al. In vivo binding of a tau imaging probe, $[11 \mathrm{C}] \mathrm{PBB} 3$, in patients with progressive supranuclear palsy. Mov Disord Off J Mov Disord Soc. 2019;34:744-54.
49. Terada T, Yokokura M, Obi T, Bunai T, Yoshikawa E, Ando I, et al. In vivo direct relation of tau pathology with neuroinflammation in early Alzheimer's disease. J Neurol. 2019;266:2186-96.

50. Perez-Soriano A, Matarazzo M, Vafai N, Shahinfard E, Miao Q, Higuchi M, et al. PBB3 binding in a patient with corticobasal syndrome. Mov Disord. 2018;33:1359-60.

51. Perez-Soriano A, Arena JE, Dinelle K, Miao Q, McKenzie J, Neilson N, et al. PBB3 imaging in Parkinsonian disorders: evidence for binding to tau and other proteins. Mov Disord. 2017;32:1016 24.

52. Schröter N, Blazhenets G, Frings L, Barkhausen C, Jost WH, Weiller C, et al. Tau imaging in the 4-repeat-tauopathies progressive supranuclear palsy and corticobasal syndrome: a $11 \mathrm{C}$ pyridinyl-butadienyl-benzothiazole 3 PET pilot study. Clin Nucl Med. 2020;45:283-7.

53. Betthauser TJ, Lao PJ, Murali D, Barnhart TE, Furumoto S, Okamura $\mathrm{N}$, et al. In vivo comparison of tau radioligands $18 \mathrm{~F}$ THK-5351 and 18F-THK-5317. J Nucl Med Off Publ Soc Nucl Med. 2017;58:996-1002.

54. Jonasson M, Wall A, Chiotis K, Saint-Aubert L, Wilking H, Sprycha M, et al. Tracer kinetic analysis of (S)- ${ }^{18} \mathrm{~F}-\mathrm{THK} 5117$ as a PET tracer for assessing tau pathology. J Nucl Med Off Publ Soc Nucl Med. 2016;57:574-81.

55. Jonasson M, Wall A, Chiotis K, Leuzy A, Eriksson J, Antoni G, et al. Optimal timing of tau pathology imaging and automatic extraction of a reference region using dynamic [18F]THK5317 PET. NeuroImage Clin. 2019;22:101681.

56. Chiotis K, Stenkrona P, Almkvist O, Stepanov V, Ferreira D, Arakawa R, et al. Dual tracer tau PET imaging reveals different molecular targets for $11 \mathrm{C}$-THK5351 and 11C-PBB3 in the Alzheimer brain. Eur J Nucl Med Mol Imaging. 2018;45:1605-17.

57. Lockhart SN, Baker SL, Okamura N, Furukawa K, Ishiki A, Furumoto S, et al. Dynamic PET measures of tau accumulation in cognitively normal older adults and Alzheimer's disease patients measured using [18F] THK-5351. PLoS One. 2016;11:e0158460.

58. Harada R, Furumoto S, Tago T, Furukawa K, Ishiki A, Tomita N, et al. Characterization of the radiolabeled metabolite of tau PET tracer 18F-THK5351. Eur J Nucl Med Mol Imaging. 2016;43: 2211-8

59. Sun X., Liang S., Fu L., Zhang X., Feng T., Li P., et al. A human brain tau PET template in MNI space for the voxel-wise analysis of Alzheimer's disease. J Neurosci Methods [Internet]. 2019;328. Available from: http://www.embase.com/search/results? subaction=viewrecord\&from=export\&id=L2002946577

60. Shidahara M, Thomas BA, Okamura N, Ibaraki M, Matsubara K, Oyama S, et al. A comparison of five partial volume correction methods for tau and amyloid PET imaging with [18F]THK5351 and [11C]PIB. Ann Nucl Med. 2017;31:563-9.

61. Hashimoto H, Kawamura K, Igarashi N, Takei M, Fujishiro T, Aihara $\mathrm{Y}$, et al. Radiosynthesis, photoisomerization, biodistribution, and metabolite analysis of $11 \mathrm{C}-\mathrm{PBB} 3$ as a clinically useful PET probe for imaging of tau pathology. J Nucl Med Off Publ Soc Nucl Med. 2014;55:1532-8.

62. Hashimoto H, Kawamura K, Takei M, Igarashi N, Fujishiro T, Shiomi S, et al. Identification of a major radiometabolite of [11C]PBB3. Nucl Med Biol. 2015;42:905-10.

63. Kimura Y, Endo H, Ichise M, Shimada H, Seki C, Ikoma Y, et al. A new method to quantify tau pathologies with (11)C-PBB3 PET using reference tissue voxels extracted from brain cortical gray matter. EJNMMI Res. 2016;6:24.

64. Kimura Y, Ichise M, Ito H, Shimada H, Ikoma Y, Seki C, et al. PET quantification of tau pathology in human brain with 11C-PBB3. J Nucl Med Off Publ Soc Nucl Med. 2015:56:1359-65.

65. Murugan NA, Chiotis K, Rodriguez-Vieitez E, Lemoine L, Ågren $\mathrm{H}$, Nordberg A. Cross-interaction of tau PET tracers with 
monoamine oxidase B: evidence from in silico modelling and in vivo imaging. Eur J Nucl Med Mol Imaging. 2019;46:1369-82.

66. Leinonen V, Rauramaa T, Johansson J, Bottelbergs A, Tesseur I, van der Ark P, et al. S-[18F]THK-5117-PET and [11C]PIB-PET imaging in idiopathic normal pressure hydrocephalus in relation to confirmed amyloid- $\beta$ plaques and tau in brain biopsies. $J$ Alzheimers Dis JAD. 2018;64:171-9.

67. Kang JM, Lee S-Y, Seo S, Jeong HJ, Woo S-H, Lee H, et al. Tau positron emission tomography using [18F]THK5351 and cerebral glucose hypometabolism in Alzheimer's disease. Neurobiol Aging. 2017;59:210-9.

68. Jeon S, Kang JM, Seo S, Jeong HJ, Funck T, Lee S-Y, et al. Topographical heterogeneity of Alzheimer's disease based on MR imaging, tau PET, and amyloid PET. Front Aging Neurosci. 2019;11:211

69. Chiotis K, Savitcheva I, Poulakis K, Saint-Aubert L, Wall A, Antoni G, et al. [18F]THK5317 imaging as a tool for predicting prospective cognitive decline in Alzheimer's disease. Mol Psychiatry. https://doi.org/10.1038/s41380-020-0815-4.

70. Lemoine L, Gillberg P-G, Svedberg M, Stepanov V, Jia Z, Huang J, et al. Comparative binding properties of the tau PET tracers THK5117, THK5351, PBB3, and T807 in postmortem Alzheimer brains. Alzheimers Res Ther. 2017;9:96.

71. Hsu J-L, Lin K-J, Hsiao I-T, Huang K-L, Liu C-H, Wu H-C, et al. The imaging features and clinical associations of a novel tau PET tracer-18F-APN1607 in Alzheimer disease. Clin Nucl Med. 2020;45:747-56.

72. Guyatt G, Oxman AD, Akl EA, Kunz R, Vist G, Brozek J, et al. GRADE guidelines: 1. Introduction-GRADE evidence profiles and summary of findings tables. J Clin Epidemiol. 2011;64:383-94.

Publisher's note Springer Nature remains neutral with regard to jurisdictional claims in published maps and institutional affiliations. 